\title{
Heat flows through the ice lithosphere of Europa
}

\author{
Javier Ruiz \\ Departamento de Geodinámica, Facultad de Ciencias Geológicas, Universidad Complutense de Madrıd, \\ Madrid, Spain \\ Semınar on Planetary Sciences, Universidad Complutense de Madrid, Madrid, Spain
}

\section{Rosa Tejero}

Departamento de Geodinámica, Facultad de Ciencias Geológicas, Universidad Complutense de Madrıd, Madrıd, Spain

\begin{abstract}
Some of the geological features on the surface of Europa's ice shell suggest the existence of a brittle lithosphere that in some regions is no more than $2 \mathrm{~km}$ thick. The surface heat flow needed to put the brittle-ductile transition in ice at a depth of $2 \mathrm{~km}$ is at least $\sim 100 \mathrm{~mW} \mathrm{~m}^{-2}$, much higher than predicted by tidal heating models for a purely conductive ice shell. A possible explanation lies in the probable existence of a convective layer beneath the brittle lithosphere, which, owing to tidal dissipation in warm ice, could contribute to higher heat flows. If a convective subsurface layer and grain size dependent flow behavior of ice are admitted, the grain size at brittle-ductile transition depth cannot be $>1 \mathrm{~mm}$, which is consistent with the required value for the onset of convection in an ice shell thinner than $20 \mathrm{~km}$.
\end{abstract}

\section{Introduction}

Galileo spacecraft observations of Europa suggest the existence of a brittle ice crust (or lithosphere) at most $-2 \mathrm{~km}$ thick, and maybe thinner locally, overlying a liquid water or ductile ice layer [Carr et al., 1998; Pappalardo et al., 1998, 1999]. Elastic and viscous models of buckling based on the spacing between possible folds in the Astypalaea Linea region give a thickness for the buckling layer of $\sim 2 \mathrm{~km}$ [Prockter and Pappalardo, 2000]. Evidence derived from the width troughs (interpreted as possible grabens) in the surroundings of Callanish, a possible impact structure, might denote a brittle-ductile transition locally as shallow as $0.5 \mathrm{~km}$ [Moore et al., 1998]. Besides this, study of ice flexion induced by a dome-type structure located close to Conamara Chaos suggests an elastic lithosphere thickness of only $\sim 0.1-0.5 \mathrm{~km}$ [Williams and Greeley, 1998].

A brittle lithosphere thickness of a few kilometers (or only hundreds of meters) seems to require heat flows of at least -100-200 $\mathrm{mW} \mathrm{m}^{-2}$ [Ruiz and Tejero, 1999; Pappalardo et al., 1999]. These heat flow values are higher than those calculated by tidal heating models [Cassen et al., 1982; Squyres et al., 1983; Ross and Schubert, 1987; Ojakangas and Stevenson, 1989]. From these it follows that Europa's mean surface heat flow higher than $\sim 50 \mathrm{~mW} \mathrm{~m}^{-2}$ (which includes tidal heating and energy dissipation by radioactive disintegration in the rocky fraction) is very difficult to justify only by conductive ice shell model.

Moreover, the presence on Europa's mottled terrain of domes, pits, and dark spots (in general named lenticulae) deforming and even disrupting preexisting plains is postulated

\section{Copyright 2000 by the American Geophysical Union.}

Paper number 1999JE001228

0148-0227/00/1999JE001228\$09.00 by Pappalardo et al. [1998] as evidence of diapirism, probably due to convective processes in the ductile ice layer and maybe in relation to the onset of convection. If lenticulae formed in relation to the onset of the convective state, then potentially convective layer thickness estimated from typical distances among those lenticulae suggests heat flows of $\sim 100$ $\mathrm{mW} \mathrm{m}{ }^{-2}$ at the time of deformation [Ruiz, 1999]. However, if lenticulae originated from a layer already actively convecting, spacing among them would not be correlated with the thickness of the layer at the onset of convection, and then, conductive heat flow through the lithosphere could be lower. Alternatively, a model has been proposed of lenticulae and chaos regions forming by melt-through of a thin ice shell, without relation to convection [Greenberg et al., 1999].

Otherwise, analysis of Europa's surface temperature made by the Galileo Photopolarimeter-Radiometer (PPR) [Spencer et al., 1999] shows the existence of thermal anomalies in the nocturnal hemisphere; these anomalies are associated with high temperature values in regions far from the equator which cannot be related to geological features or albedo distribution. These anomalies could be caused by endogenic heat flows as high as $1100 \mathrm{~mW} \mathrm{~m}^{-2}$ locally [Spencer et al., 1999]. Because of the strong discrepancy with theoretical estimations mentioned above, Spencer et al. [1999] favor an explanation based on variations in surface thermal inertia, maybe in relation to different textures of superficial material.

Taking into account the brittle-ductile transition depth suggested by geological and structural features, a detailed quantitative approach to heat flow through Europa's ice lithosphere, by means of the comparison of brittle and ductile strength in the ice shell, is proposed in this work. Although this analysis does not take into account the possible existence of a convective subsurface layer, or that of an internal ocean, some implications of these questions can be followed from the results. 


\section{Brittle-Ductile Transition and Heat Flow}

Assuming preexisting planes of fractures of all orientations, in the absence of pore fluid pressure (as expected in an icy satellite), the brittle strength is given by [Jaeger and Cook, 1979]

$$
\left(\sigma_{1}-\sigma_{3}\right)_{b}=2\left(\mu \sigma_{3}+S_{0}\right)\left[\left(\mu^{2}+1\right)^{1 / 2}+\mu\right],
$$

where $\sigma_{1}$ and $\sigma_{3}$ are maximum and minimum principal stresses, $S_{0}$ is the material cohesion, and $\mu$ is the friction coefficient. For water ice, under normal stress conditions of $\sigma_{n}<20 \mathrm{MPa}$, which are suitable for the ice lithosphere of Europa, $S_{0}=1 \mathrm{MPa}$ and $\mu=0.55$ [Beeman et al., 1988]. For the sake of clarity, we write $\left(\mu^{2}+1\right)^{1 / 2}+\mu=B$. Assuming one principal vertical stress axis, in pure horizontal compression

$$
\sigma_{3}=\rho g z
$$

where $\rho$ is density above the considered level (in Europa's shell, water ice density, $\left.930 \mathrm{~kg} \mathrm{~m}^{-3}\right), \mathrm{g}$ is gravity acceleration, assumed here as the surface value $\left(1.31 \mathrm{~m} \mathrm{~s}^{-2}\right)$, and $\mathrm{z}$ is the depth; substituting (2) into (1) the following is obtained:

$$
\left(\sigma_{1}-\sigma_{3}\right)_{b}=2 B\left(\mu \rho g z+S_{0}\right) \text {. }
$$

Likewise, in pure horizontal tension

$$
\sigma_{3}=\rho g z-\left(\sigma_{1}-\sigma_{3}\right)_{b}
$$

substituting (4) into (1) gives

$$
\left(\sigma_{1}-\sigma_{3}\right)_{b}=2 B\left(\mu \rho g z+S_{0}\right) /(2 \mu B+1) .
$$

Ductile strength can be estimated from steady state creep law. Here we will consider two deformation mechanisms: grain boundary sliding (grain size dependent) and dislocation creep (grain size independent). Constitutive law for creep has the form

$$
\left(\sigma_{1}-\sigma_{3}\right)_{d}=\left(\cdot \varepsilon d^{\prime \prime} / A\right)^{1 / n} \exp \left(Q / n R T_{z}\right),
$$

where $\varepsilon$ is the strain rate, $d$ is the grain size, $Q$ is the activation energy for creep, $R=8.3144 \mathrm{~J} \mathrm{~mol}^{-1} \mathrm{~K}^{-1}$ is the gas constant, $T_{\mathrm{z}}$ is the temperature at the considered depth, and $A$, $p$, and $n$ are constants. Grain boundary sliding has been postulated as the dominant ductile mechanism of deformation for Europa's ice shell conditions [Pappalardo et al., 1998; McKınnon, 1999].

Following Goldsby and Kohlstedt [1997], values of constants are $Q=49 \mathrm{~kJ} \mathrm{~mol}^{-1}, p=1.4$, and $n=1.8$, and $A$ estimation gives $51.86 \mathrm{MPa}^{-n} \mathrm{~mm}^{\prime \prime} \mathrm{s}^{-1}$. The calculations have been carried out considering a grain size of $0.1 \mathrm{~mm}$, according to surface observations [Geissler et al., 1998], and grain sizes of 1 and $10 \mathrm{~mm}$, according to a possible increase of this parameter with depth.

If we allow for larger grain sizes, then dislocation creep $(p=0)$ should be considered. Parameters for water ice dislocation creep have been determined by Durham et al. $[1992,1997]$ for planetary conditions. For ice $I$ in regime $A$ $(T>240 \mathrm{~K}), A=10^{118} \mathrm{MPa}^{-n} \mathrm{~s}^{-1}, n=4$, and $Q=91 \mathrm{~kJ} \mathrm{~mol}^{-1}$. For ice I in regime $\mathrm{B}(T<240 \mathrm{~K}), A=10^{51} \mathrm{MPa}^{-n} \mathrm{~s}^{-1}, n=4$, and $Q=61 \mathrm{~kJ} \mathrm{~mol}^{-1}$.
Taking into account colatitude, longitude, and stresses induced by tides, strain rate in Europa's ice shell has been estimated at $\sim 1.2-2.5 \times 10^{-10} \mathrm{~s}^{-1}$ [Ojakangas and Stevenson, 1989]. We can use an average value of $2 \times 10^{-10} \mathrm{~s}^{-1}$ in the calculations without introducing any appreciable difference in final estimations. Other processes that could have caused deformation in Europa's ice shell have associated lower strain rates [Pappalardo et al., 1998], and therefore they will not be considered here.

According to Fourier's law, the lithospheric depthtemperature profile is governed by conductive heat transport,

$$
F=k(d T / d z)
$$

where $F$ is the vertical heat flow through the lithosphere (assumed constant) and $k$ is the water ice thermal conductivity. The conductivity $k$ is a function of temperature and for water ice is $k=k_{0} / T$, where $k_{0}=567 \mathrm{~W} \mathrm{~m}^{-1}$ [Klinger, 1980]. The temperature at any depth $z$ is given by

$$
T_{z}=T_{s} \exp \left(F z / k_{0}\right),
$$

where $T$, is the lithosphere top temperature. In (8) we have assumed that in a conductive regime ice lithosphere is heated from below, which is reasonable, because, though in the same ice shell heat is dissipated by tidal friction, this effect depends on temperature and is clearly dominated by the deepest and hottest part of ice [Ojakangas and Stevenson, 1989].

The $T$, values significantly influence the estimations. Here, In order to determine the temperature profile, two different temperature values at the top of the ice lithosphere have been considered: $100 \mathrm{~K}$, thought to be representative of Europa's surface mean temperature [Ojakangas and Stevenson, 1989], and $130 \mathrm{~K}$, a value that is normally assigned to the base of a possible isolating regolith layer on the surface [Shoemaker et al.. 1982] or one that could be reached if a solid-state greenhouse in the most superficial part of the ice exists [Matson and Brown, 1989].

Brittle-ductile transition represents the depth where the ice shell has the same brittle and ductile strength:

$$
\left(\sigma_{1}-\sigma_{3}\right)_{1}(z)=\left(\sigma_{1}-\sigma_{3}\right)_{d}(z)
$$

Taking $h$ as the brittle-ductile transition depth and substituting $z$ for $h$ in (9), the heat tlow through the icy lithosphere is given by

$$
F=\left(k_{0} / h\right) \ln \left\{\left(Q / R T_{1}\right) / \ln \left[\left(\sigma_{1}-\sigma_{3}\right)_{l}{ }^{n}\left(A / \cdot \varepsilon d^{p}\right)\right]\right\},
$$

where $\left(\sigma_{1}-\sigma_{3}\right)_{b}$ is the brittle strength when $z=h$.

Figure 1 shows the brittle and ductile strength plots for $T$, $=130 \mathrm{~K}$; strength envelopes are computed for grain boundary slidıng and dislocatıon creep. Figure 2 represents surface heat flow values in terms of brittle-ductile transition depth, $T_{\text {, }}$ and $d$. The influence of grain size on $F$ values obtained for grain boundary sliding is shown in Figure 3.

\section{Discussion}

The brittle-ductile transition, in essence, marks the depth at which temperatures are high enough to permit ductile deformation to dominate. In this regard, the Figure 1 and 2 plots clearly show that heat flows implied by a brittle ice layer 


$$
\mathrm{Ts}=130 \mathrm{~K} ; \mathrm{d}=0.1 \mathrm{~mm}
$$

a)

$$
\sigma_{1} \cdot \sigma_{3}(\mathrm{MPa})
$$

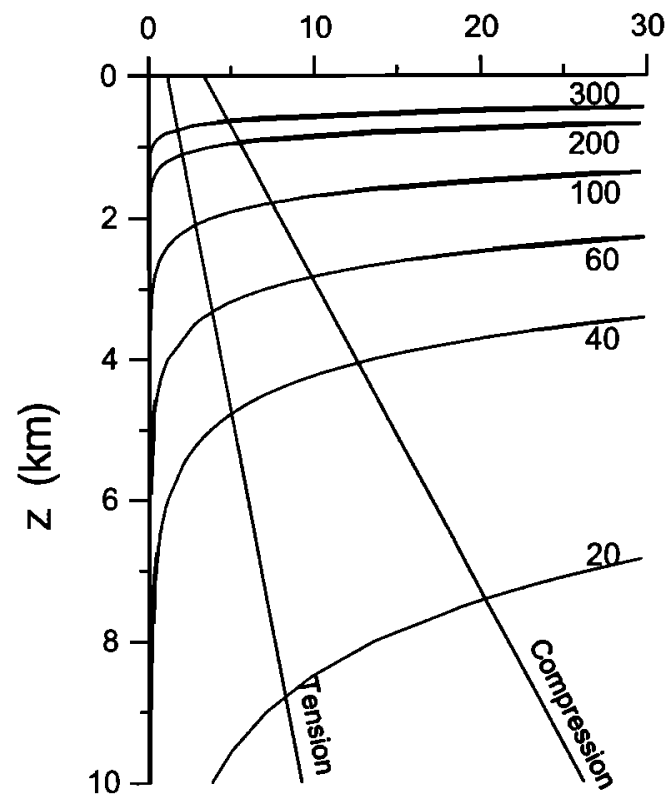

$$
\mathrm{Ts}=130 \mathrm{~K} ; \mathrm{d}=10 \mathrm{~mm}
$$

c)

$$
\sigma_{1}-\sigma_{3} \quad(\mathrm{MPa})
$$

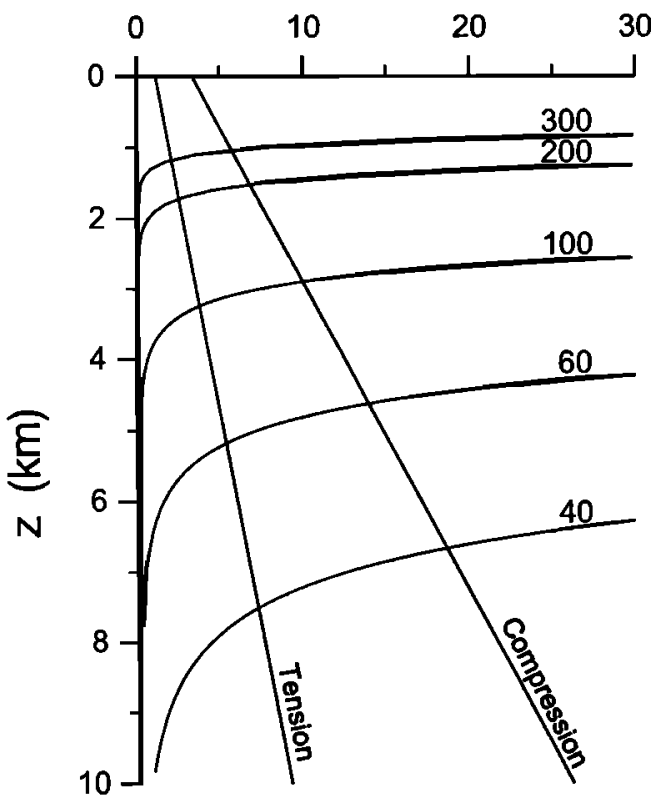

$$
\mathrm{Ts}=130 \mathrm{~K} ; \mathrm{d}=1 \mathrm{~mm}
$$

b)

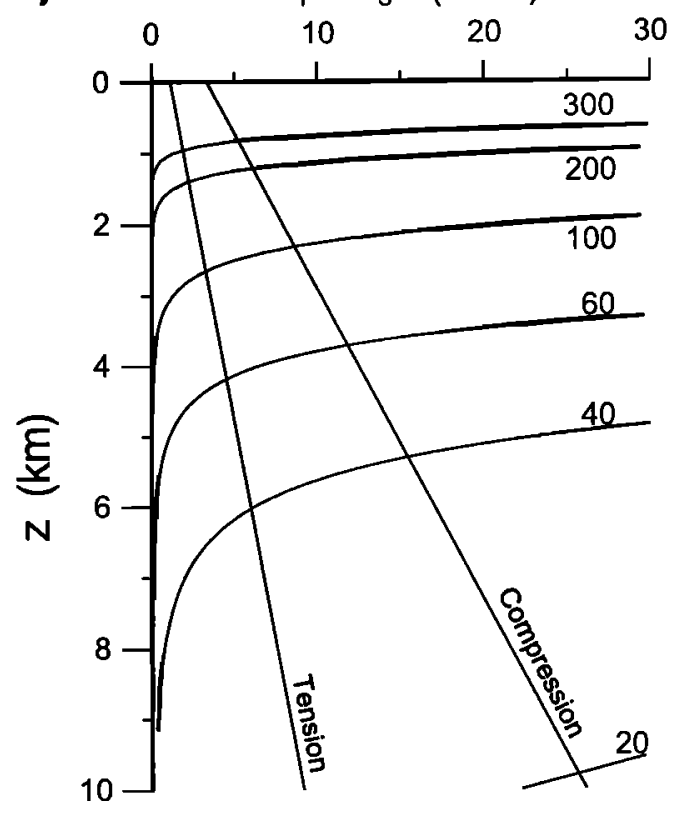

$$
\mathrm{Ts}=130 \mathrm{~K}
$$

d)

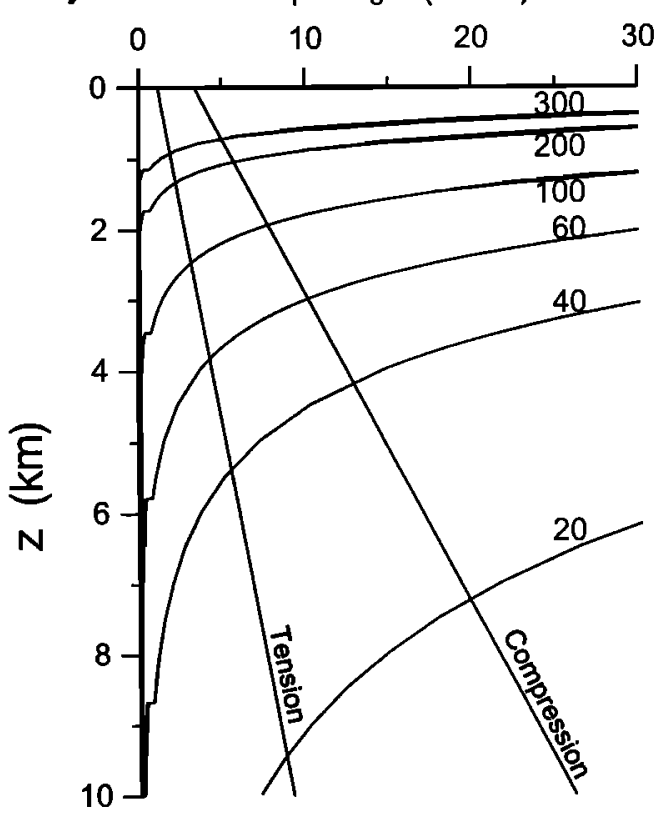

Figure 1. Europa's ice shell strength envelopes calculated for $T_{s}=130 \mathrm{~K}$. Here $d$ is grain size and $z$ is depth. Every envelope presents a brittle-ductile transition depth for different heat flow values $\left(\mathrm{mW} \mathrm{m}^{-2}\right)$. Figures 1 alc are calculated assuming grain boundary sliding, and Figure 1d is calculated assuming dislocation creep. Brittle strength depends on tectonic regime.

of $\sim 2 \mathrm{~km}$ thickness [Carr et al., 1998; Pappalardo et al., 1998, 1999] are much higher than those obtained in tida] heating models. Taking into account the uncertainties derived from surface effective temperature, possible grain size dependence and range, and differences between tectonic regimes, heat flow values might be between approximately 80 and $250 \mathrm{~mW} \mathrm{~m}^{-2}$. If we consider grain boundary sliding or dislocation creep as the dominant mechanism of ductile deformation, the results do not vary significantly. Moreover, brittle ice thickness lower than $2 \mathrm{~km}$ or, in the case of grain 

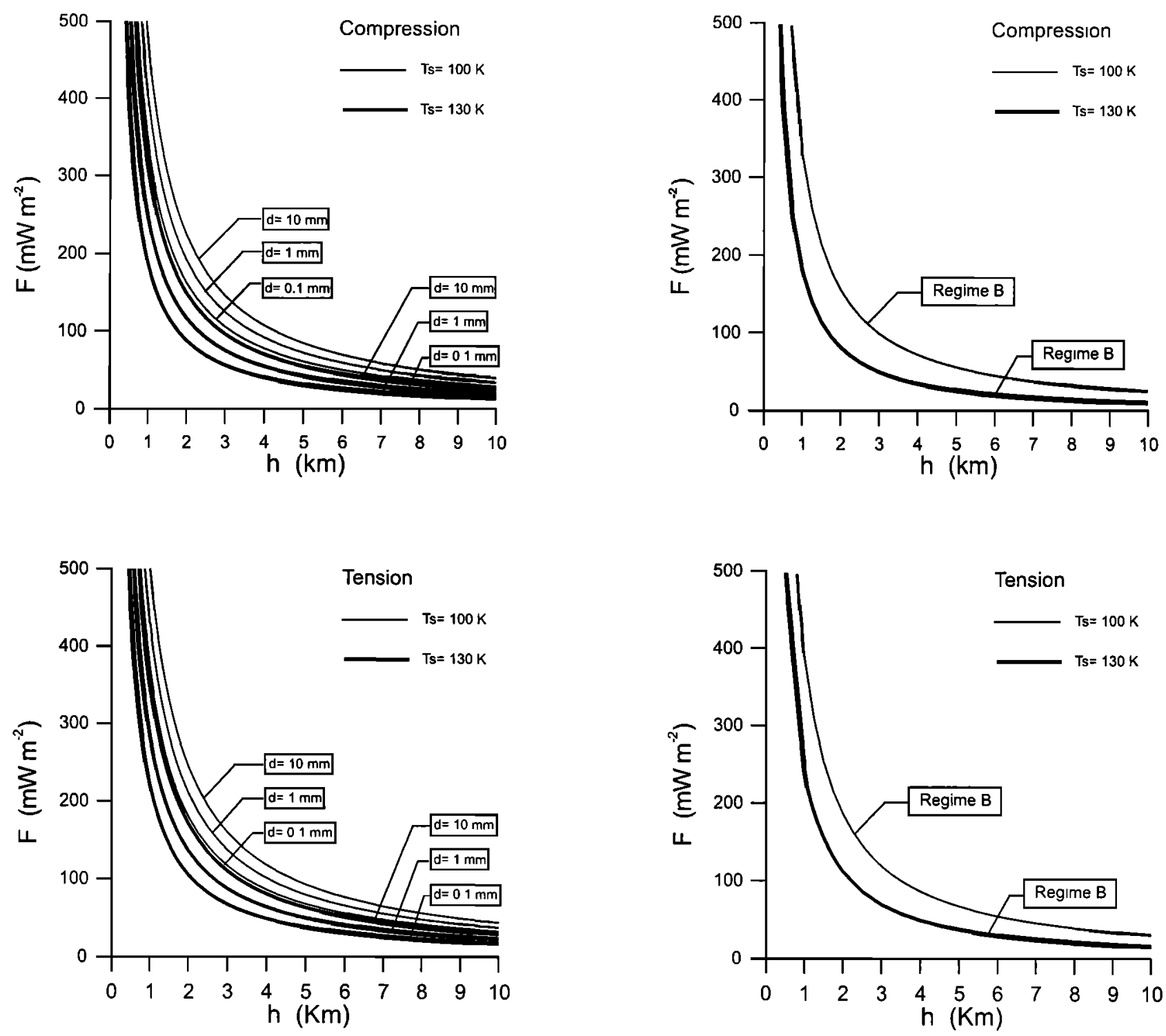

a) Grain boundary sliding

b) Dislocation creep

Figure 2. Heat flow variations in terms of brittle-ductile transition depth for grain boundary sliding and dislocation creep. Here $h$ is depth, $T_{s}$ is surface temperature, and $d$ is grain size. Teclonic regimes characterized by compression and tension stress fields are considered.

boundary sliding, larger grain size implies higher heat flows. A brittle-ductile transition as shallow as $0.5 \mathrm{~km}$ requires heat flows of at least $\sim 400-500 \mathrm{~mW} \mathrm{~m}^{-2}$, values that seem too high, even if we consider them as a local phenomenon. In this way, the possible heat flows of even $1100 \mathrm{~mW} \mathrm{~m}^{-2}$, discussed (but not supported) by Spencer et al. [1999] after observations of the Galileo Photopolarimeter-Radiometer, are still higher; although they would be adjusted with a predicted higher tidal heating in higher latitudes [Ojakangas and Stevenson, 1989], other explanations have been suggested [Spencer et al., 1999].

An explanation of heat flow discrepancies in Europa may be that high heat flows are a local phenomenon and that the lithosphere thickness changes from one region to another [Head et al., 1999]. In this case, the average heat flow could be more in keeping with previously expected values. The shell thıckness could also change with time, and so those high heat flow values could not be a current phenomenon.

Another explanation would be that grain size in brittle lithosphere was $<0.1 \mathrm{~mm}$. so ductile strength for grain boundary sliding would be reduced and $F$ values would be lower. However, as $0.1 \mathrm{~mm}$ is precisely the estimated grain size for the surface, this alternative does not seem very reasonable. The existence of grain sizes $<1 \mathrm{~mm}$ is difficult to explain if there are not impurities which limit the crystal growth [McKinnon, 1999]. Moreover, a heat flow of $\sim 60 \mathrm{~mW}$ $\mathrm{m}^{-2}$, still high, would correspond to grain sizes as unrealistic as $0.01 \mathrm{~mm}$.

As it has been mentioned, lenticulae have been interpreted as evidence of diapirism induced from a subsurface layer in convection [Pappalardo et al., 1998]. At the onset of convection, the Nusselt number (convective and conductive 


\section{a) COMPRESSION}

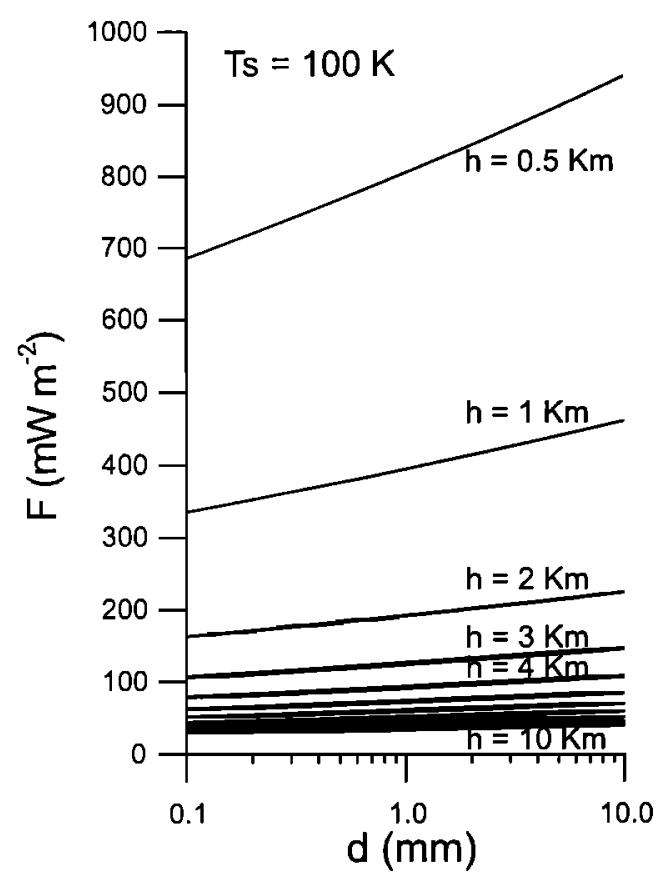

b ) TENSION

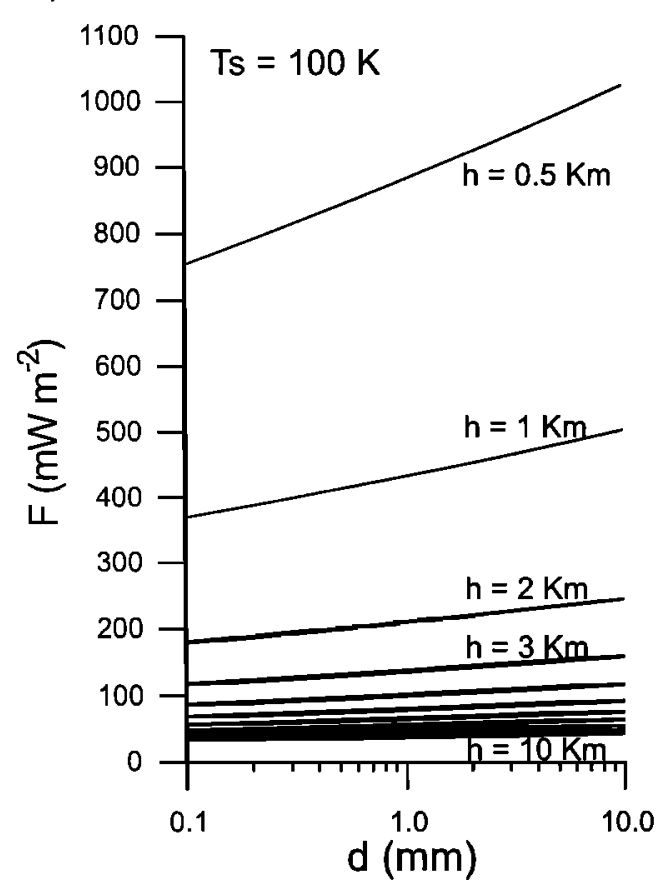

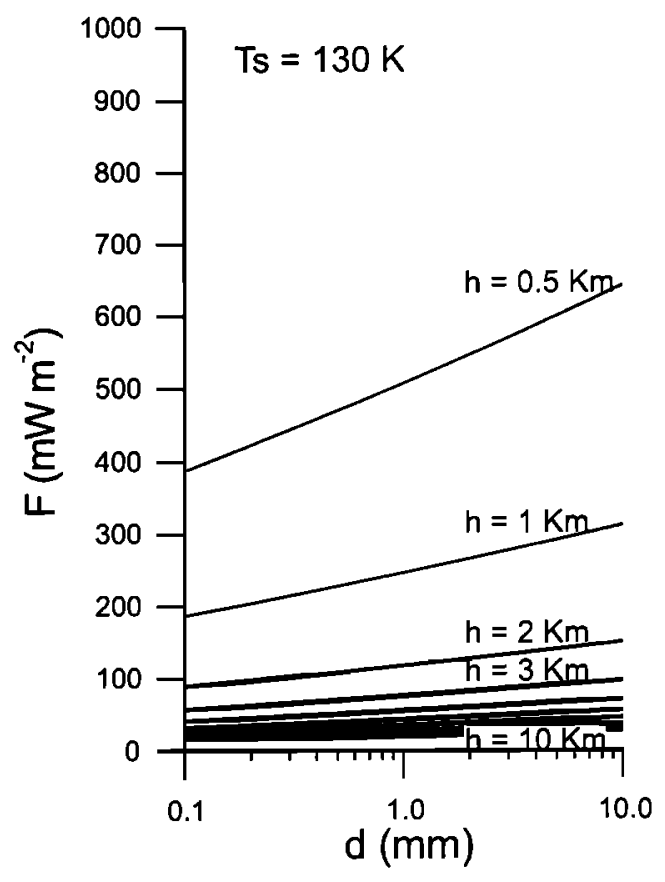

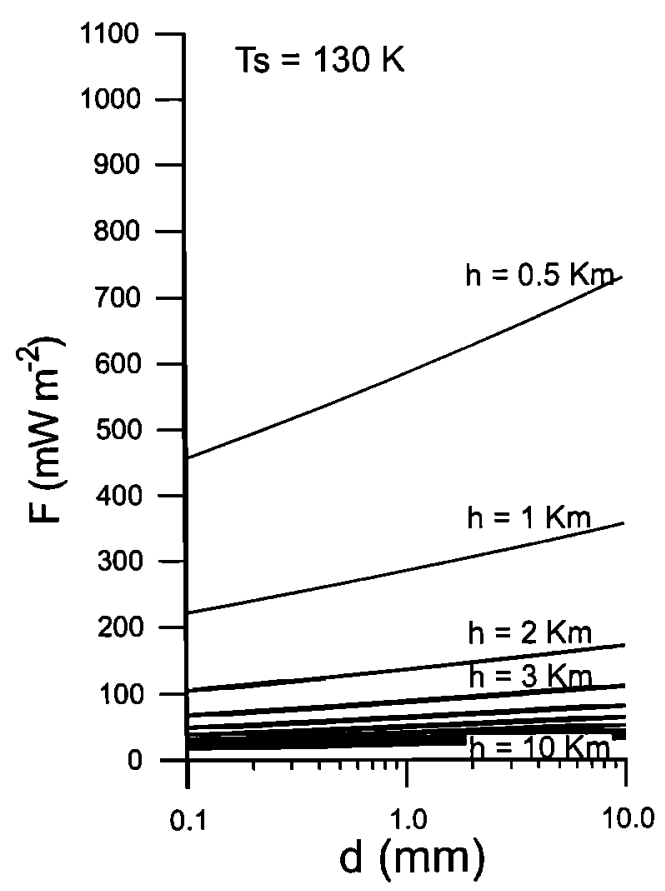

Figure 3. Grain size influence in the calculation of heat flow, in terms of $h$, for grain boundary sliding.

heat flow ratio) increases until $~ 1.5$ [McKinnon, 1999], which involves a heat flow $\sim 50 \%$ higher than in the previous situation, with all of the ice shell in a conductive state. If convection is well established, the Nusselt number can be higher, and so can the dissipated heat flow. In this situation, the lithosphere thickness is rebalanced with these higher heat flows, and it is thinner than it would be in a solely conductive shell. On the other hand, because of tidal heating in the convective layer, with a high average temperature (adiabatic) of $\sim 260 \mathrm{~K}$, an internal ocean could probably avoid freezing [McKinnon, 1999].

From Ojakangas and Stevenson's [1989] work, for a 260 $\mathrm{K}$ temperature, a mean volumetric rate of tidal dissipation $\mathrm{H}$ can be calculated: $\sim 0.003-0.01 \mathrm{~mW} \mathrm{~m}^{-3}$. So, the average contribution of a convective ice layer in adiabatic conditions (in an energetic equilibrium situation [Turcotte and Schubert, 1982] ) to the total heat flow is

$$
F_{\text {conv }}=H b \text {, }
$$




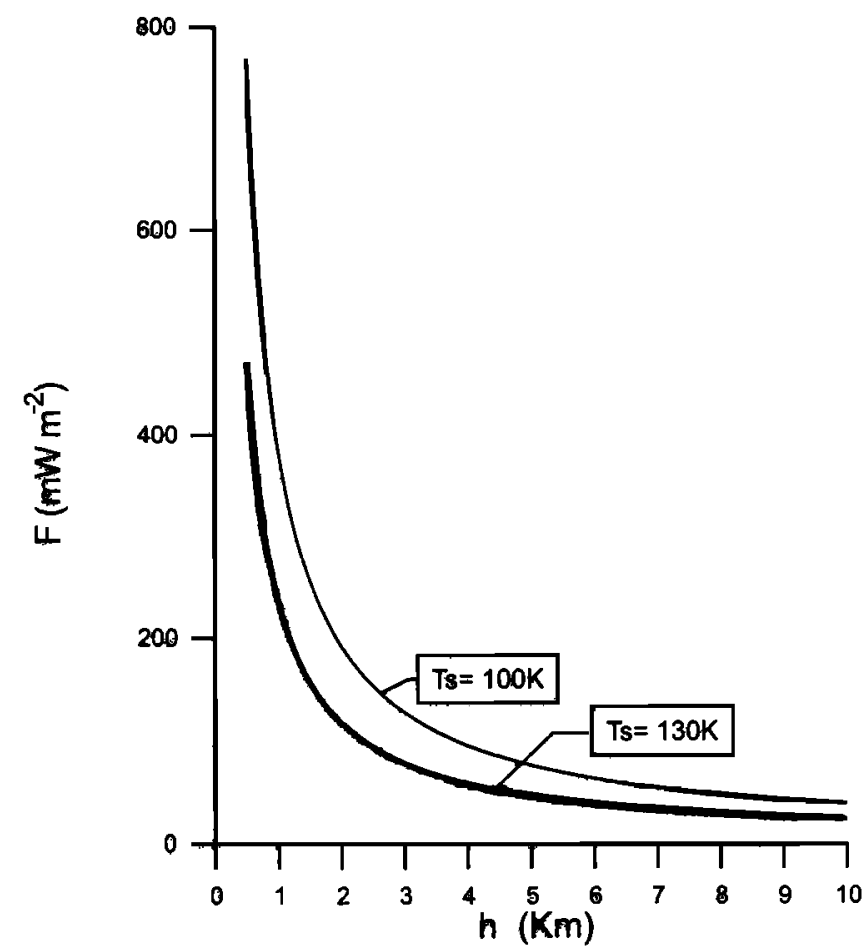

Figure 4. Maximum heat flow (in terms of $h$ ) if the existence of a convective subsurface layer in Europa's ice shell is admitted, taking into account the extreme situation in which the rheological lithosphere base coincides with the stagnant lid base of the convective system.

where $b$ is the thickness of the adiabatic ice layer, which can be taken as the thickness of the convective layer. If we take a core heat flow of $-20 \mathrm{~mW} \mathrm{~m}^{-2}$, due to a tidal and radiogenic dissipation [Squyres et al., 1983; Ross and Schubert, 1987], we would need a thickness of the convective layer of at least $\sim 6 \mathrm{~km}$ to justify the heat flow values obtained in this work, according to the typical spacing of lenticulae at surface, $\sim 5-20$ $\mathrm{km}$, which would be roughly twice the thickness of the layer in convection [Pappalardo et al., 1998]. In this setting, higher heat flows involve higher $b$ values. However, it does not seem reasonable to generate (or dissipate) in this way $F$ values of $\sim 1 \mathrm{~W} \mathrm{~m}^{-2}$.

If it is admitted that grain boundary sliding is the most important ductile deformation mechanism in Europa's ice shell [Pappalardo et al., 1998; McKinnon, 1999], and also that a convective subsurface layer exist, we can establish approximate upper limits to heat flow and grain size at the brittle-ductile transition level. Pappalardo et al. [1998] find a temperature of $197 \mathrm{~K}$ for the top of the layer in convection as a function of activation energy of creep deformation by grain boundary sliding. Though the base of the stagnant lid of the convective system does not necessarily have any reason to be the same as the base of the lithosphere or rigid lid [McKinnon, 1998, 1999], the maximum heat flow corresponds precisely to the situation in which both coincide, and it comes from

$$
F_{\mathrm{mux}}=\left(k_{0} / h\right) \ln \left(T_{\mathrm{bsl}} / T_{s}\right),
$$

where $T_{\text {bsl }}$ is the temperature at the base of the stagnant lid (temperature in the top of the layer in convection). In Figure 4 we show $F_{\max }$ in terms of $h$. If we take $T_{z}=T_{a} \exp \left(F h / k_{0}\right)=$ $T_{\mathrm{bsl}}$ in (9), the maximum grain size is

$$
d_{\max }=\left[\left(\sigma_{1}-\sigma_{3}\right)_{b}{ }^{n}(A / \cdot \varepsilon) \exp \left(-Q / R T_{\mathrm{hs}}\right)\right]^{1 / p}
$$

$\left(\left(\sigma_{1}-\sigma_{3}\right)_{b}\right.$ is the brittle strength when $\left.z=h\right)$, from which it results that $d_{\max } \approx 0.3-1.1 \mathrm{~mm}$ for $h=2 \mathrm{~km}$, depending on the tectonic regime. For $h<2, \mathrm{~km} d_{\max }$ is lower. These values have an approximate character, as brittle-ductile transition depth depends on tectonic regime and strain rate. They suggest that the grain size, at the same level as the brittleductile transition at least, can be, in general, lower than $1 \mathrm{~mm}$. In this sense, for the onset of convection in a floating ice shell on Europa, a grain size precisely $<1 \mathrm{~mm}$ is needed if the total thickness of ice is under $-20 \mathrm{~km}$, and $<0.3 \mathrm{~mm}$ is needed for a shell thinner than $\sim 10 \mathrm{~km}$ [McKinnon, 1999]. In this way the theoretical Europan ice thickness at the onset of convection is poorly determined; recently, ice thicknesses of $\sim 3-10 \mathrm{~km}$ [Pappalardo et al., 1998] and 6-23 km [McKinnon, 1999] have been proposed in relation to, precisely, the ice viscosity dependence on ice grain size for grain boundary sliding.

\section{Conclusions}

Estimations of brittle and ductile strengths in a brittle lithosphere a few kilometers thick show that heat flows in Europa's surface must reach at least $\sim 100 \mathrm{~mW} \mathrm{~m}^{-2}$. Although a thin brittle lithosphere could be a regional characteristic, the classical tidal heating models, made for ice shells in a conductive state, are difficult to reconcile with these results. Furthermore, high heat flows could be supported by the discovery of thermal anomalies in the nocturne hemisphere of the satellite. The tidal energy dissipation efficiency in warm ice in the adiabatic core of a convective layer may constitute a solution to the problem.

Finally, from impact crater scarcity, the age of Europa's surface can be estimated to within $-10^{7}$ years [Carr et al., 1998; Zahnle et al., 1998; Pappalardo et al., 1999], so it is very probable that this Jovian satellite continues to be active today. On the other hand, lenticulae correspond to some youngest superficial features of Europa [Pappalardo et al., 1998; Head et al., 1999; Prockter et al., 1999], so it seems very reasonable that the convective system continues to work at present. If it were so, energy liberation by tidal heating in warm ice in the layer in convection might allow the maintenance of a liquid water internal ocean a few kilometers or a few tens of kilometers under the surface.

Acknowledgments. We thank F. Anguita, R. T. Pappalardo, N. Sánchez, and A. Torices for their comments and suggestions and two anonymous referees, who helped to improve the final version of this paper.

\section{References}

Beeman, M., W. B. Durham, and S. H. Kirby, Friction of ice, $J$. Geophys. Res., 93, 7625-7633, 1988.

Carr, M. H., et al., Evidence for a subsurface ocean on Europa, Nature, 391, 363-365, 1998.

Cassen, P. M., S. J. Peale, and R. T. Reynolds, Structure and thermal evolution of the Galilean satellites, in Satellites of Jupiter, edited by D. Morrison, pp. 93-128, Univ. of Ariz. Press, Tucson, 1982.

Durham, W. B., S. H. Kirby, and L. A. Stern, Effects of dispersed particulates on the rheology of water ice at planetary conditions, J. Geophys. Res., 97, 20,883-20,897, 1992. 
Durham, W. B., S. H. Kirby, and L. A. Stern, Creep of water ices at planetary conditions: A compilation, J. Geophys. Res., 102, 16,293-16,302, 1997.

Geıssler, P. E . et al., Evolution of lineaments on Europa: Clues from Galıleo Multsspectral Imaging observations, Icarus, 135, 107126. 1998

Goldsby, D. L., and D. L. Kohlstedt, Grain boundary sliding in finegrained Ice I, Scri. Mater., 37, 1399-1406, 1997.

Greenberg, R., G. V. Hoppa, B. R. Tufts, P. Geissler, J. Riley, and S. Kadel, Chaos on Europa, Icarus, 14I, 263-286, 1999.

Head, J. W., R. T. Pappalardo, L. M. Prockter, N. A. Spaun, G. C. Collins, R. Greeley, J. E. Klemaszewski, R. Sullivan, C Chapman, and the Galıleo SSI Team, Europa: Recent geological history from Galileo observations, Lunar Planet. Sci. [CD-ROM], $X X X$, abstract 1404, 1999.

Klinger, $J$, Influence of a phase transition of the ice on the heat and mass balance of comets, Scrence, 209, 271-272, 1980.

Jaeger, J. C., and N. G. W. Cook, Fundamentals of Rock Mechanics, 3rd ed., 593 pp.. Chapman and Hall, New York, 1979.

Matson. D L., and R. H Brown, Solid-state greenhouses and their Implications for icy satellites, Icarus, 77, 67-81, 1989.

McKinnon, W. B., Geodynamics of icy satellites, in Solar System lies, edited by B. Schmitt, C. De Bergh, and M. Festou, pp. 525550, Kluwer Acad., Norwell, Mass., 1998.

McKınnon, W. B., Convective instability in Europa's floating ice shell, Geophys Res. Lett., 26, 951-954, 1999.

Moore, J. M., et al., Large Impact features on Europa: Results of the Galıleo Nominal Mission, Icarus, 135, 127-145, 1998.

Ojakangas, G. W and D. J Stevenson, Thermal state of an ice shell on Europa, Icarus, 81, 220-241, 1989.

Pappalardo, R. T., et al., Geological evidence for solid-state convection in Europa's ice shell, Nature, 391, 365-368, 1998.

Pappalardo, R. T., et al., Does Europa have a subsurface ocean? Evaluation of the geological evidence, J. Geophys. Res., 104, 24,015-24,055, 1999

Prockter, L. M., and R. T. Pappalardo, Evidence for regional-scale folds on Europa, Lunar Planer. Sci. [CD-ROM], XXXI, abstract $1182,2000$.
Prockter, L. M., A. M. Antman, R. T. Pappalardo, J. W. Head, and G. C. Colluns, Europa: Stratigraphy and geological history of the anti-Jovian region from Galileo E14 solid-state imaging data, $J$. Geophys. Res., 104, 16,531-16,540, 1999.

Ross, M. N., and G. Schubert, Tidal heating in an internal ocean model of Europa, Nature, 325, 133-134, 1987.

Ruiz, J., Onset of convection, heat flow and thickness of the Europa's ice shell, Earth Moon Planets, 77, 99-104, 1999.

Ruiz, J., and R Tejero 1999. Heat flow and brittle-ductıle transition in the ice shell of Europa, Lunar Planet. Sci. [CD-ROM], XXX, abstract 1031, 1999.

Shoemaker, E M., B K. Lucchita, D. E. Wilhelms, J. B. Plescia, and S W. Squyres, The geology of Ganymede, in Satellites of Jupiter, edited by D. Morrison, pp. 435-520, Univ. of Ariz. Press, Tucson, 1982.

Spencer, J. R., L K Tamppari, T. Z. Martin, and L. D. Travis, Temperatures on Europa from Galileo PhotopolarimeterRadiometer: Nighttime thermal anomalies, Science, 284, 1514$1516,1999$.

Squyres, S. W., R. T. Reynolds, P. M. Cassen, and S. J. Peale, Liquid water and active resurfacing on Europa, Nature, 301, 225-226, 1983.

Turcotte, D L., and G. Schubert, Geodynamics: Applications of Continuum Physics to Geological Problems, 450 pp., John Wiley, New York, 1982.

Williams, K. K., and R. Greeley, Estimates of ice thickness in the Conamara Chaos region of Europa, Geophys. Res. Lett., 25, 4273 4276, 1998.

Zahnle, K., L. Dones, and H. F. Levison, Cratering rates on the Galilean satellites, Icarus, 136, 202-230, 1998.

J. Ruiz and R. Tejero, Departamento de Geodinámica, Facultad de Ciencias Geológicas, Universidad Complutense de Madrid, 28040 Madrid, Spain. (jaruiz@eucmax.sim.ucm.es)

(Received December 19, 1999; revised August 23, 2000; accepted September 12, 2000) 